\title{
Anti-Idiotype scFv Localizes an Autoepitope in the Globular Domain of C1q
}

\author{
Nadezhda Todorova ${ }^{1}$, Miroslav Rangelov ${ }^{2}$, Vanya Bogoeva ${ }^{3}{ }^{\oplus}$, Vishnya Stoyanova ${ }^{4}$, Anna Yordanova ${ }^{3}$, \\ Ginka Nikolova ${ }^{5}{ }^{\circ}$, Hristo Georgiev ${ }^{6}\left(\mathbb{D}\right.$, Daniela Dimitrova ${ }^{7}$, Safa Mohedin ${ }^{5}$, Katerina Stoyanova ${ }^{5}$ \\ and Ivanka Tsacheva ${ }^{5, *}$ (D)
}

check for updates

Citation: Todorova, N.; Rangelov, M.; Bogoeva, V.; Stoyanova, V.; Yordanova, A.; Nikolova, G.; Georgiev, H.; Dimitrova, D.; Mohedin, S.; Stoyanova, K.; et al. Anti-Idiotype scFv Localizes an Autoepitope in the Globular Domain of C1q. Int. J. Mol. Sci. 2021, 22, 8288. https://doi.org/10.3390/ijms22158288

Academic Editor: Gian

Marco Ghiggeri

Received: 5 July 2021

Accepted: 29 July 2021

Published: 1 August 2021

Publisher's Note: MDPI stays neutral with regard to jurisdictional claims in published maps and institutional affiliations.

Copyright: (C) 2021 by the authors. Licensee MDPI, Basel, Switzerland. This article is an open access article distributed under the terms and conditions of the Creative Commons Attribution (CC BY) license (https:// creativecommons.org/licenses/by/ $4.0 /)$
1 Institute of Biodiversity and Ecosystem Research (IBER), Bulgarian Academy of Sciences, Yurii Gagarin Str. Bl. 2 1113 Sofia, Bulgaria; nadeshda@abv.bg

2 Institute of Organic Chemistry with Centre of Phytochemistry (IOCCP), Bulgarian Academy of Sciences, Acad. G. Bonchev Str. B1. 9, 1113 Sofia, Bulgaria; marangelov@gmail.com

3 Institute of Molecular Biology, Bulgarian Academy of Sciences, Acad. G. Bonchev Str. Bl. 21, 1113 Sofia, Bulgaria; vanya.bogoeva@gmail.com (V.B.); anna_gurova@bio21.bas.bg (A.Y.)

4 Department of Chemistry, Biochemistry, Physiology and Pathophysiology,

Medical Faculty of Sofia University, 1 Kozyak Str., 1407 Sofia, Bulgaria; vishnya_stoyanova@abv.bg

5 Department of Biochemistry, Faculty of Biology, Sofia University, 8 D. Tsankov Str., 1164 Sofia, Bulgaria; ginka.nikolova@biofac.uni-sofia.bg (G.N.); sofibg@hotmail.com (S.M.); katerina_stoyanova_@abv.bg (K.S.)

6 Institute of Immunology, Hannover Medical School, Carl-Neuberg Str. B1.1, 30625 Hannover, Germany; georgiev.hristo@mh-hannover.de

7 Institute of Biophysics and Biomedical Engineering, Department of Electroinduced and Adhesive Properties, Bulgarian Academy of Sciences, Acad. G. Bonchev Street, Bl. 21, 1113 Sofia, Bulgaria; daniadim@yahoo.com

* Correspondence: itsacheva@biofac.uni-sofia.bg

Abstract: We addressed the issue of $\mathrm{C} 1 \mathrm{q}$ autoantigenicity by studying the structural features of the autoepitopes recognized by the polyclonal anti-C1q antibodies present in Lupus Nephritis (LN) sera. We used six fractions of anti-C1q as antigens and selected anti-idiotypic scFv antibodies from the phage library "Griffin.1". The monoclonal scFv A1 was the most potent inhibitor of the recognition of $\mathrm{C} 1 \mathrm{q}$ and its fragments ghA, ghB and ghC, comprising the globular domain $\mathrm{gC1q}$, by the lupus autoantibodies. It was sequenced and in silico folded by molecular dynamics into a 3D structure. The generated 3D model of A1 elucidated CDR similarity to the apical region of gC1q, thus mapping indirectly for the first time a globular autoepitope of $\mathrm{C} 1 \mathrm{q}$. The $\mathrm{V}_{\mathrm{H}} \mathrm{CDR} 2$ of A1 mimicked the ghA sequence GSEAD suggested as a cross-epitope between anti-DNA and anti-C1q antibodies. Other potential inhibitors of the recognition of $\mathrm{C} 1 \mathrm{q}$ by the $\mathrm{LN}$ autoantibodies among the selected recombinant antibodies were the monoclonal scFv F6, F9 and A12.

Keywords: C1q; globular autoepitopes; scFv; anti-DNA

\section{Introduction}

$\mathrm{C} 1 \mathrm{q}$, the recognition molecule of the classical complement pathway, appears as an autoantigen in several human autoimmune disorders, most notably in systemic lupus erythematosus (SLE) [1-4]. SLE is characterized by a broad spectrum of autoantibody specificities, some of which are directed against nuclear components and C1q. Anti-dsDNA autoantibodies, the hallmark of SLE, are hypothesized as a result of defective removal of apoptotic material, eventually resulting in an immune response to these normally sequestered autoantigens [5]. The anti-C1q autoantibodies, which closely follow the appearance of anti-dsDNA, are hypothesized as a result of conformational changes in C1q due to immobilization and exposure of neo-epitopes [6-8], underlain by increased hydrophobicity [9] or/and as a result of post-translational modifications [10].

Anti-C1q autoantibodies are associated with lupus nephritis (LN), a clinical condition in more than $30 \%$ of SLE patients [11]. LN follows a course of exacerbations and remissions 
and more than $25 \%$ of LN patients experience multiple episodes of active nephritis at increased risk of progressing to end stage renal disease [12].

The anti-C1q autoantibodies are known to be polyclonal high affinity IgG molecules, produced in an antigen-driven process [13-15]. The complex structure of C1q provides two types of functional domains-the $\mathrm{N}$-terminal collagen-like region (CLR) and the C-terminal globular heads ghA, ghB and ghC, associated in a globular domain (gC1q). Autoepitopes for the anti-C1q antibodies have been detected on both of them [15-18]; however, so far only a linear epitope in CLR has been localized [19]. The localization of epitopes in $\mathrm{gClq}$ is challenging due to the fact that ghA, ghB and ghC are structurally and functionally independent modules [20]. The heterotrimeric organization of $\mathrm{gClq}$ offers functional flexibility and possible versatility of epitopes for recognition by the autoantibodies. The autoantigenic behavior of $\mathrm{C} 1 \mathrm{q}$ is reflected by specific features of its 3D structure. Accordingly, the localization of $\mathrm{C} 1 \mathrm{q}$ autoepitopes and studying their structural features would largely contribute to our understanding of $\mathrm{C} 1 \mathrm{q}$ autoantigenicity and would suggest the molecular events leading to the generation of autoantibodies to C1q.

In the present study, we addressed the issue of $\mathrm{C} 1 \mathrm{q}$ autoantigenicity by studying the structural features of $\mathrm{C} 1 \mathrm{q}$ autoepitopes that are recognized by human polyclonal anti-C1q autoantibodies present in the sera of LN patients. In our previous research, we have found that $\mathrm{LN}$ sera were positive for anti-C1q antibodies that recognized different combinations of $\mathrm{C} 1 \mathrm{q}$ domains and their smaller fragments, e.g., CLR and the globular fragments of $\mathrm{A}, \mathrm{B}$ and $\mathrm{C}$ chains comprising $\mathrm{gClq}-\mathrm{ghA}$, ghB and ghC, along with the intact molecule. Presumably, these sera contained anti-C1q antibodies with different epitope specificities [15]. Consequently, we combined the sera with similar epitope specificities and used the subgroups of anti-C1q antibodies, isolated from them, to generate anti-idiotype $\mathrm{scFv}$ antibodies which we applied as tools to localize $\mathrm{C} 1 \mathrm{q}$ autoepitopes. For some scFv antibodies, we detected inhibitory activity on the recognition of immobilized C1q by human LN autoantibodies using immunosorbent and spectrofluorometric analyses. By molecular dynamics simulation we generated a 3D model of the most potent inhibitor-the monoclonal scFv A1, which mapped to the apical region of gC1q comprising parts of ghA, ghB and ghC.

\section{Results}

\subsection{Generation of Anti-Idyotipic scFv}

Based on our previous study of the epitope specificity of the polyclonal autoantibodies to $\mathrm{C} 1 \mathrm{q}$ from LN sera [15], we classified the analysed sera into six groups according to their positivity for the used test-antigens: intact C1q, CLR and ghA, ghB and ghC as representatives of gC1q. Sera that were positive for the intact $\mathrm{C} 1 \mathrm{q}$ (pooled in group $\mathrm{A}$ ) became the source of anti-C1q designated fraction A (Table 1). Sera positive for ghC (pooled in group $D$ ) yielded autoantibodies designated fraction $\mathrm{D}$. Sera that were positive for combinations of test-antigens were pooled in other groups as follows: group $B(C 1 q+C L R)$, group $C$ $(\mathrm{C} 1 \mathrm{q}+\mathrm{CLR}+\mathrm{ghC})$, group $\mathrm{E}(\mathrm{CLR}+\mathrm{ghA} / \mathrm{ghB}+\mathrm{ghC})$ and group $\mathrm{F}(\mathrm{ghA} / \mathrm{ghB}+\mathrm{ghC})$, and became sources of anti-C1q fractions B, C, E and F, respectively. Antibodies from each group of pooled sera were affinity purified and used as antigens for the selection of monoclonal scFv, part of which we expected to be anti-idiotypic and therefore potential structural analogues of $\mathrm{C} 1 \mathrm{q}$ autoepitopes. The anti-C1q fractions $\mathrm{A}, \mathrm{B}$ and $\mathrm{C}$ were extracted by incubation of respective pooled sera diluted in PBS containing $0.75 \mathrm{M} \mathrm{NaCl}$ with immobilized intact $\mathrm{C} 1 \mathrm{q}$, as it was the common test-antigen for these groups, while anti-C1q fractions D, E and F were extracted likewise on immobilized ghC, the common test-antigen for the rest sera groups. High ionic strength was needed to inhibit the IgG molecules that tended to interact with $\mathrm{Clq}$ via $\mathrm{Fc}$ and to allow the interaction of $\operatorname{IgG}$ molecules via their $\mathrm{Fab}$, thus ensuring their anti-C1q specificity. The affinity procedure was performed before every round of selection and the presence of anti-C1q IgG antibodies was routinely checked by immunoblotting against the whole set of test-antigens (data not shown). Interestingly, eluted anti-C1q fractions $(\mathrm{A}-\mathrm{C}$ ) gave a strong signal not only to intact $\mathrm{C} 1 \mathrm{q}$ but also to ghA, 
while eluted anti-ghC fractions (D-F) gave comparable signals in their binding to intact C1q, ghA, ghB and ghC. After three rounds of selection of Griffin.1 library [21], we rescued 68 monoclonal scFv antibodies and tested them for antigen binding-10 clones selected for anti-C1q fraction A (A1 to A10), 13 clones selected for fraction B (B1 to B12 and A12), 12 clones selected for fraction $C$ ( $C 1$ to $C 12), 11$ clones selected for fraction D (D1 to D11), 11 clones selected for fraction E (E1 to E11) and 11 clones selected for fraction F (F1 to F11). Twenty-one of them were specific for their respective antigens (Table 2). Phage particles from each specific scFv clone were transferred to non-suppressor strain E.coli HB2151 and expressed in soluble form. The antigen specificity of the soluble scFv was confirmed by the detection of both scFv tags- $\mathrm{C}$ myc and His (Table 3). We ruled out the scFv clones which were specific for isotypic epitopes by using a negative control of pooled human IgG from healthy donors, thus selecting 14 anti-idiotypic scFv clones.

Table 1. Distribution of autoimmune sera into six groups according to the epitope specificities detected for them. Bolded are the common antigens for groups A, B and C (intact C1q) and the common test-antigen for groups $\mathrm{D}, \mathrm{E}$ and $\mathrm{F}$ (ghC).

\begin{tabular}{cc}
\hline $\begin{array}{c}\text { Sera Group/ } \\
\text { Anti-C1q Fraction }\end{array}$ & Sera, Positive for Autoantibodies against: \\
\hline A & intact C1q \\
\hline B & intact C1q and CLR \\
\hline C & intact C1q and CLR and ghC \\
\hline D & ghC \\
\hline E & CLR and/or ghA and/or ghB and ghC \\
\hline F & ghA and/or ghB and ghC
\end{tabular}

Table 2. Immunoblotting of 68 rescued phage displayed scFv clones against anti-C1q fractions (A-F). Nitrocellulose membranes, coated with anti-C1q (A-F) and blocked with $0.5 \%$ Tween 20 , were incubated with $10^{12}$ recombinant phage displayed scFv in TPBS from each clone. Bound phage was detected by anti-M13-HRP (1:2000 in TPBS) and DAB. The (+) and $(-)$ refer to positive and negative signals, respectively. The (+/-) refers to a weak signal which was not considered positive.

\begin{tabular}{|c|c|c|c|c|c|c|c|c|c|c|c|c|}
\hline Anti-C1q Fraction & 1 & 2 & 3 & 4 & 5 & 6 & 7 & 8 & 9 & 10 & 11 & 12 \\
\hline A & + & - & + & - & + & - & - & + & + & - & & + \\
\hline B & - & + & - & - & - & + & - & - & + & - & - & - \\
\hline C & + & - & - & - & + & - & - & - & + & - & - & - \\
\hline $\mathrm{D}$ & - & + & - & - & - & - & + & + & - & - & - & \\
\hline $\mathrm{E}$ & + & - & - & - & - & $+/-$ & - & - & $+/-$ & - & - & \\
\hline $\mathrm{F}$ & - & - & $+/-$ & - & - & + & - & - & + & - & - & \\
\hline
\end{tabular}

\subsection{ELISA Analysis for Selection of Inhibitory scFv}

We analysed the selected anti-idiotypic clones as potential inhibitors on the recognition of immobilized C1q by the LN autoantibodies. The large-scale analysis was done with partially purified $\mathrm{scFv}$ by ammonium sulfate precipitation. The source of autoantibodies was affinity purified IgG from a pool of all analysed $L N$ sera, designated $\operatorname{IgG}_{\mathrm{LN}}$. The analysis was performed by competitive ELISA, with increasing amounts of the competitors. We found four clones with inhibitory capacity: A1 (anti-fraction A), A12 (anti-fraction B), F6 and F9 (anti-fraction F) (Figure 1, Table S1). Clone A1 showed the greatest extent of inhibition by the smallest amount of introduced competitor. Consequently, it was expressed at a large scale and purified by affinity $\mathrm{Ni}+$ chromatography. The purified scFv A1 exhibited $47 \%$ inhibition on the recognition of immobilized C1q by the LN autoantibodies (Figure 2A). Furthermore, A1 inhibited to a greater extent the recognition of ghA, ghB and 
ghC by the same autoantibodies (Figure 2B). This data suggested that $\mathrm{A} 1$ was structurally analogous to an autoepitope located in gC1q. The inhibition of all three globular heads by A1 indicated that they all contributed to the formation of a globular autoepitope, thus suggesting its conformational nature.

Table 3. Dot blotting of 21 monoclonal soluble scFv against anti-C1q fractions (A-F). Nitrocellulose membranes, coated with anti-C1q (A-F) and blocked with $0.5 \%$ Tween 20, were incubated with $2 \mathrm{~mL}$ of scFv-containing SN from each clone. Bound phage was detected by anti-C myc (9E10 mouse MoAb, 1:2000 in TPBS) and anti-mouse IgG-AP (1:2000 in TPBS), or alternatively by His-Probe-HRP (1:3 000 in TPBS). The anti-idiotypic clones are marked with $\left(^{*}\right)$. Human IgG was used as a control for isotypic epitopes, e.g., as a negative control. The $(+)$ and (-) refer to positive and negative signals, respectively. The $(+/-)$ refers to a weak signal which was not considered positive.

\begin{tabular}{|c|c|c|c|c|c|}
\hline \multicolumn{2}{|c|}{ Antigen for Screening } & \multirow{2}{*}{$\begin{array}{l}\text { C myc } \\
+/-\end{array}$} & \multirow{2}{*}{$\begin{array}{c}\text { His-Tag } \\
+\end{array}$} & \multirow{2}{*}{$\begin{array}{c}\text { Human IgGfrom Healthy Donors } \\
-\end{array}$} & \multirow{2}{*}{$\begin{array}{c}\text { Potentially } \\
\text { Anti-Idiotypic scFv } \\
*\end{array}$} \\
\hline \multirow{5}{*}{ Anti-C1q } & A1 & & & & \\
\hline & A3 & $+1-$ & + & - & * \\
\hline & A5 & $+/-$ & + & - & * \\
\hline & A8 & $+/-$ & + & - & $*$ \\
\hline & A9 & $+1-$ & + & - & * \\
\hline \multirow{4}{*}{$\begin{array}{l}\text { Anti-C1q and } \\
\text { Anti-CLR }\end{array}$} & B2 & $+/-$ & + & - & $*$ \\
\hline & B6 & $+/-$ & + & - & * \\
\hline & B9 & $+1-$ & + & $+/-$ & \\
\hline & A12 & $+/-$ & + & - & * \\
\hline \multirow{3}{*}{$\begin{array}{l}\text { Anti-C1q and } \\
\text { Anti-CLR and } \\
\text { Anti-ghC }\end{array}$} & $\mathrm{C} 1$ & $+1-$ & + & $+/-$ & \\
\hline & $\mathrm{C} 5$ & $+1-$ & + & - & * \\
\hline & C9 & $+/-$ & + & - & $*$ \\
\hline \multirow{3}{*}{ Anti-ghC } & D2 & $+1-$ & + & $+/-$ & \\
\hline & D7 & $+/-$ & + & $+/-$ & \\
\hline & D8 & $+/-$ & + & - & * \\
\hline \multirow{3}{*}{$\begin{array}{l}\text { Anti-CLR and/or } \\
\text { Anti-ghA and/or } \\
\text { Anti-ghB and } \\
\text { Anti-ghC }\end{array}$} & E1 & $+/-$ & + & - & * \\
\hline & E6 & $+1-$ & + & $+1-$ & \\
\hline & E9 & $+/-$ & + & $+/-$ & \\
\hline \multirow{3}{*}{$\begin{array}{l}\text { Anti-ghA and /or } \\
\text { Anti-ghB and } \\
\text { Anti-ghC }\end{array}$} & F3 & $+/-$ & & $+/-$ & \\
\hline & F6 & $+1-$ & + & - & $*$ \\
\hline & F9 & $+/-$ & + & - & $*$ \\
\hline
\end{tabular}

\subsection{Fluorescence Spectroscopy Analysis}

The inhibitory capacity of scFv A1 was verified by fluorescent spectroscopy. LN autoantibodies complexed with scFv A1 inhibited the binding of C1q, ghA, ghB and ghC, respectively, when applied in increasing concentrations.

The inhibitory capacity of scFv A1 was clearly detected by its ability to block the recognition of C1q by LN autoantibodies (Figure 3). Extrinsic fluorescence of the hydrophobic dye ANS was used for the experimental study in order to avoid the intrinsic protein fluorescence. In Figure 3A, the ANS spectrum is shown (1, lowest spectrum), LN autoantibodies complexed with scFv A1 (2, upper spectrum) and C1q (3-6) in increasing concentrations $(0.03 \mu \mathrm{M}-0.3 \mu \mathrm{M})$. The blocking effect is shown by $\mathrm{C} 1 \mathrm{q}$ spectra that have overlapped the spectrum of the complexed autoantibodies with scFv A1. 


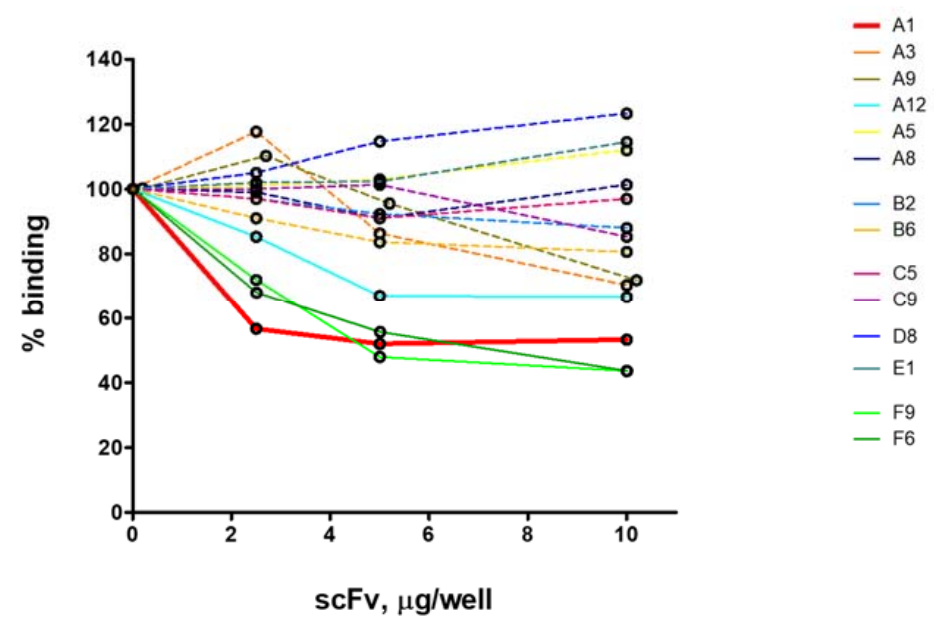

Figure 1. ELISA analysis of the inhibitory capacity of the anti-idiotypic monoclonal scFv antibodies on the recognition of immobilized $\mathrm{C} 1 \mathrm{q}$ by $\mathrm{IgG}_{\mathrm{LN}}$.

A

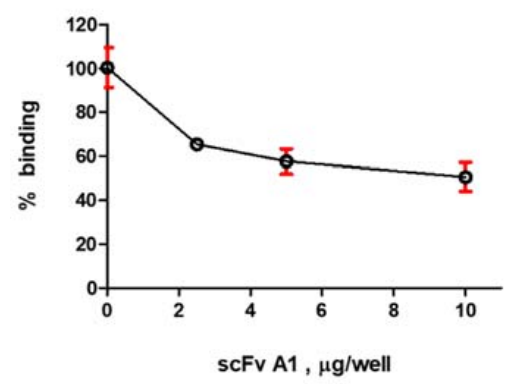

B

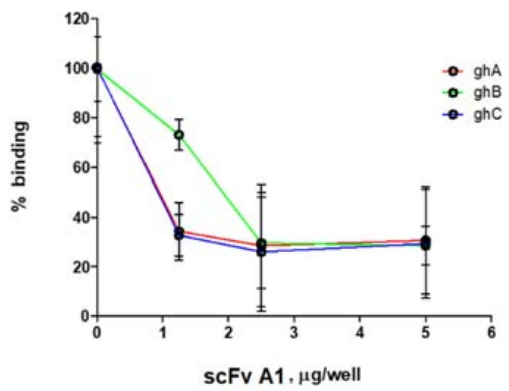

Figure 2. (A) ELISA analysis of the inhibitory capacity of $\mathrm{scFv} \mathrm{A} 1$ on the recognition of immobilized C1q by $\operatorname{IgG}_{\mathrm{LN}}$. Error bars (red) indicate mean \pm SD. (B) ELISA analysis of the inhibitory capacity of $\mathrm{scFv} \mathrm{A} 1$ on the recognition of immobilized ghA, ghB and ghC by $\operatorname{IgG}_{\mathrm{LN}}$. Error bars (black) indicate mean \pm SD.

A

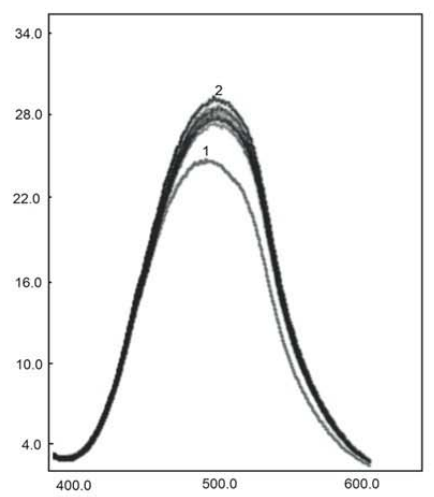

B

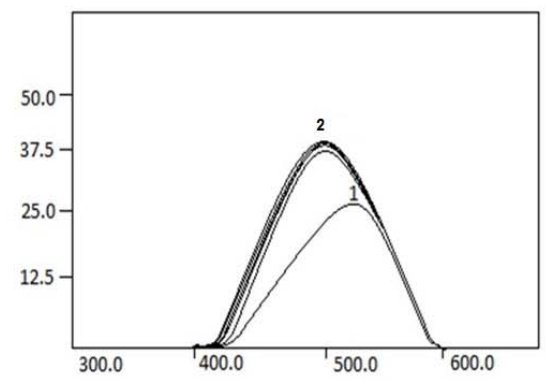

c

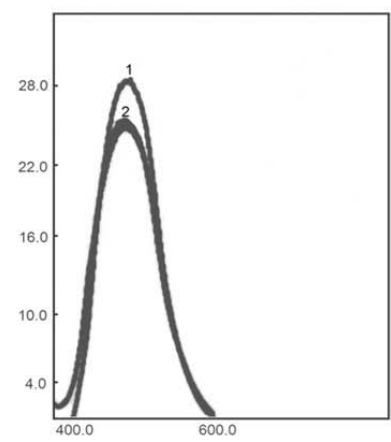

Figure 3. (A) Fluorescence spectra, showing ANS (1, lowest spectrum), LN autoantibodies complexed with scFv A1 (2, upper spectra) and $\mathrm{C} 1 \mathrm{q}$ in increasing concentrations $(0.03 \mu \mathrm{M}-0.3 \mu \mathrm{M}$, overlapping the spectrum of the complex autoantibodies and scFv A1). (B) Fluorescence spectra, showing ANS (1, lowest spectrum), LN autoantibodies complexed with scFvA1 (2, upper spectra) and ghA in increasing concentrations $(0.03 \mu \mathrm{M}-0.3 \mu \mathrm{M}$, overlapping the spectrum of the complexed autoantibodies with scFv A1). (C) Fluorescence spectra, showing ANS (1, upper spectrum), LN autoantibodies complexed with scFvA1 (2, lower spectra) and ghC/ghB in increasing concentrations $(0.03 \mu \mathrm{M}-0.3 \mu \mathrm{M}$, overlapping the spectrum of the complexed autoantibodies with scFv A1). 
Similar to $\mathrm{C} 1 \mathrm{q}$ binding, we performed experiments with ghA, ghB and ghC fragments. The results showed inhibitory effects of the studied proteins (Figure $3 B, C$ ). Experimental data with ghB and ghC fragments had similar results, showing inhibition of scFv A1 upon the ghB and ghC.

\subsection{Sequence Analysis of the Selected scFv A1}

The scFv A1 sequence, containing only antibody coding parts, was further analysed. EMBOSS sequence tools revealed only 1 ORF, standard codon table, stretched on the whole length of the $\mathrm{scFv}$ (223 amino acids).

The sequence was compared to the human immunoglobulin germ-line V-gene sequences and the CDR prediction analysis was performed with VBASE2 platform [22]. Heavy chain V-segment are of the IGHV4 family, while the light chain is classified as IGKV1 subgroup. No J-segment is discovered in the $\mathrm{V}_{\mathrm{L}}$; however, the junction in $\mathrm{V}_{\mathrm{H}}$ participated as expected in the CDR3 formation.

\subsection{ScFv A1 Homology Model}

The sequence analysis of scFv A1 further continued with generating its homology model (Figure 4) in order to detect possible molecular similarity with the C1q molecule. Structural comparison of C1q globular heads and scFv A1 was performed on $\mathrm{V}_{\mathrm{H}}$, as $\mathrm{V}_{\mathrm{H}}$ CDR3 was expected to exhibit the greatest degree of variety and, accordingly, to have a distinctive role in antigen recognition. The comparison was based on the assumption that the reacting amino acids were situated in flexible loops of both structures, rather than the structurally compact and rigid beta-sheet structures. CDR1, 2 and 3 were compared loop by loop with C1q separate chains loops. The similarity is based on the polarity of the amino acids (Figure 6). The backbone is analysed only as amino acid side chain (R) polarity due to the mobility of $\mathrm{R}$ in solution, and not as a chain position in the loop. A snapshot of the described procedure is depicted in Figure 5.

A

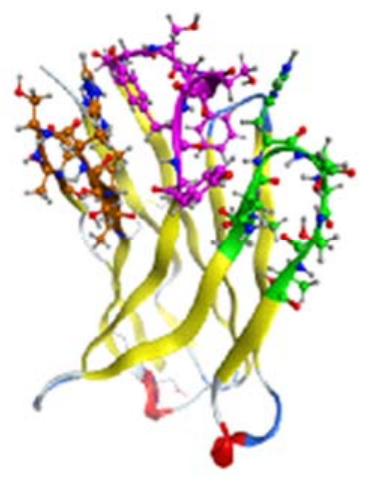

8

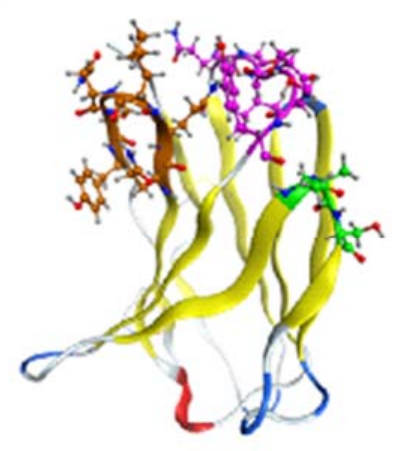

Figure 4. Representation of the generated model backbone of scFv A1 H-chain (A) and scFv A1 L-chain (B) in color coding (yellow beta structure, blue for loops). CDR1, 2 and 3 amino acids are depicted in magenta, green and brown, respectively. The revealed CDRs of H-chain are CDR1 (GGSFSGYY), CDR2 (INHSGST) and CDR3 (ARSHSAA). The revealed CDRs of L-chain are CDR1 (QGISSY), CDR2 (AAS) and CDR3 (QQLNSY).

All CDR loops have their most similar residues in regions involved in the $\mathrm{C} 1 \mathrm{q}$ interfaces, namely between C- and D-, G-/H- and E-/F- loops according to Gaboriaud et al. [23]. Notably, these parts are not conserved in $\mathrm{C} 1 \mathrm{q}$ and similar proteins, but rather a substantial variability in the residues is found at the interfaces, thus eliminating occasional likeness. Based on the loop similarity model, all of the three CDR regions of scFv VH have analogs in the apex of native gC1q chains (Figure 7) that are known to exhibit differences in their surface patterns, with respect to both charged and hydrophobic residues. These findings indicate the sites on the exposed surface of $\mathrm{gClq}$, which are important for the engagement 
of lupus antibodies and either contribute to or lie in close proximity with detected C1q parts, similar to scFv A1 CDRs. The mimicry of scFv A1 to parts of all globular heads ghA, $\mathrm{ghB}$ and ghC reveals possible involvement of all of them in the interaction with the lupus autoantibodies and is consistent with the inhibitory activity of scFv A1 towards all three globular heads, thus confirming the conformational nature of the mimicked autoepitope.

A

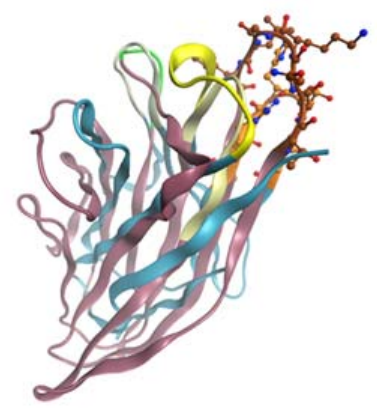

B

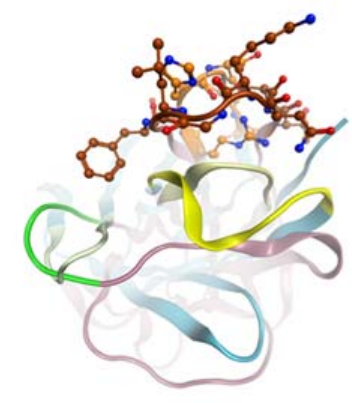

Figure 5. Side view (A) and top view (B) of $\mathrm{V}_{\mathrm{H}}$ model (blue ribbons for frame regions) aligned on ghA of $\mathrm{C} 1 \mathrm{q}$ (pink ribbons); CDR1, CDR2 and CDR3 of scFvA1 and ghA interface regions are presented in green, yellow and brown, respectively, with different color intensity in both chains.

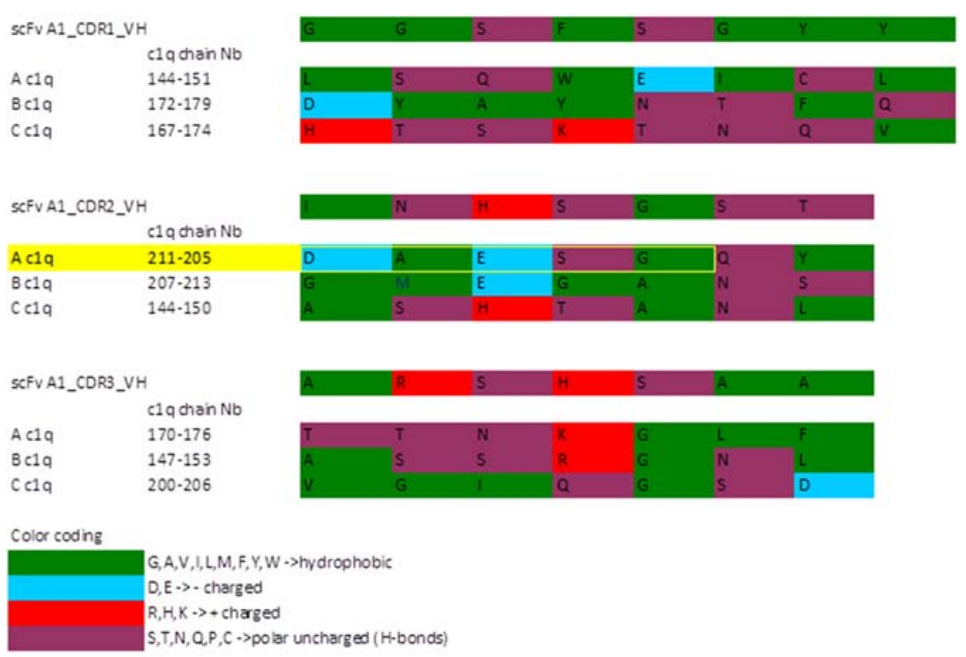

Figure 6. The optimal correlation of R polarity between C1q globular heads and CDR loops of $\mathrm{H}$ chain scFv A1. The suggested DNA/C1q cross-reacting autoepitope GSEADV by Franchin et al. [24] from the ghA sequence is marked in yellow.

A

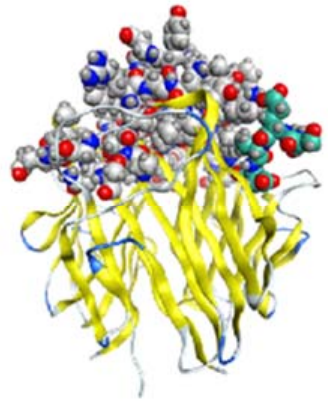

B

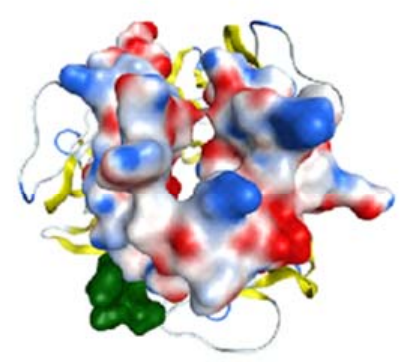

Figure 7. gC1q (yellow ribbons) parts, identified as structurally similar to scFvA1 $\mathrm{V}_{\mathrm{H}} \mathrm{CDR} 1,2$ and 3 (grey, blue and red spheres); (A) Side view, where the cross-reactive DNA/C1q autoepitope ([24]; see Discussion) is clearly seen in light green colour. (B) Top view of the molecular surface with presented electrostatic potential, again in green is depicted the cross-reactive DNA/C1q autoepitope. 


\section{Discussion}

We generated scFv A1 as anti-idiotypic antibody to a fraction of LN anti-C1q autoantibodies and it turned out to be a potent inhibitor of the recognition of $\mathrm{C} 1 \mathrm{q}$, ghA, ghB and ghC by the $\mathrm{LN}$ autoantibodies. The generated 3D model of A1 sequence elucidated CDR similarity to the apical region of $\mathrm{gClq}$, thus mapping indirectly for the first time a globular autoepitope of $\mathrm{C} 1 \mathrm{q}$. The similar regions are situated in the three globular heads, suggesting that the globular autoepitope may consist of a number of linear stretches, thus being a conformational one. This is consistent with the fact that the anti-C1q, named fraction A, were extracted from LN sera, positive only for the intact C1q. Presumably, each of the globular heads is of low affinity when binding the LN autoantibodies and, consequently, none of them was detected as individual test-antigen.

Our experimental data suggest also that there are other potential inhibitors of the recognition of $\mathrm{C} 1 \mathrm{q}$ by the $\mathrm{LN}$ autoantibodies among the selected recombinant antibodies, namely the monoclonal scFv F6, F9 and A12 (Figure 1). The clones, F6 and F9, are likely to mimic an autoepitope within $\mathrm{gClq}$ given that the anti-C1q fraction $\mathrm{F}$ (Table 1) contains autoantibodies specific for ghA and/or ghB and ghC. The clone A12 is anti-idiotypic to anti-C1q fraction B containing autoantibodies specific for C1q and CLR, consequently it is expected to mimic an epitope within CLR.

Anti-C1q autoantibodies have been researched for a long time [25]. The focus of the research for the best part of that time was the CLR of C1q because the anti-CLR autoantibodies strongly correlated with disease activity [26-31]. However, the detection of autoantibodies binding gC1q opened up new possibilities for an insight into $\mathrm{C} 1 \mathrm{q}$ autoantigenicity. Anti-gC1q antibodies, unlike anti-CLR, were found to be prevalent in non-active LN patients [15]. Moreover, again unlike anti-CLR, we detected these autoantibodies in high titers among healthy humans [32]. Collectively, these data brought us to the assumption that the appearance of anti-gC1q antibodies would act more as a trigger rather than a mediator of the clinical outbreak of C1q-associated autoimmunity. As C1q is an extracellular antigen, its autoantigenicity does not fit in the concept of the resonance hypothesis [33], according to which the tissue targeting generates additional autoantigens that fuel further autoimmune response. It has been reported that autoantibodies develop before the clinical onset $[34,35]$ and among the first specificities to appear are anti-dsDNA antibodies which also exhibit the highest sensitivity for diagnosing SLE. The loop similarity of scFv A1 and gC1q revealed that $\mathrm{V}_{\mathrm{H}} \mathrm{CDR} 2$ of $\mathrm{scFv} \mathrm{A} 1$ mimics the ghA sequence GSEAD, which was suggested as a cross-epitope between anti-DNA and anti-C1q antibodies [24]. We hypothesized that the cross-reacting anti-dsDNA could be the antibodies dragging C1q initially into the context of autoimmune response by turning it into an autoantigen. This assumption agrees with the observations that, chronologically, anti-C1q autoantibodies appear after anti-dsDNA autoantibodies [34,35]. It is also likely that the binding of these autoantibodies with gC1q would introduce the first conformational changes that could lead to a subsequent exposure of neo-epitope in distant parts of the molecule.

Previously, we have found that conformational disturbance within ghB affected the degree of autoantigenicity of the whole $\mathrm{C} 1 \mathrm{q}$ molecule [9]. If our hypothesis is supported by further analysis, it would mean that anti-gC1q autoantibodies are the first to appear in the autoimmune setting involving $\mathrm{C} 1 \mathrm{q}$ as autoantigen. This type of initiating factor is unlikely to act alone but rather in combination with others. Recently, Csorba et al. reported that anti-C1q antibodies specific for the major linear epitope of CLR are cross-reacting with EBNA-1 (Epstein-Barr virus nuclear antigen 1)-derived peptide, implying that anti-C1q in SLE could be induced through molecular mimicry by EBV [36].

Interestingly, the BLAST analysis of the scFv A1 sequence revealed $91 \%$ similarity in the CDR regions of DQ201310.1, $\mathrm{H3} \mathrm{Ab}$, which is an anti-EBV antibody [37]. It recognizes one of the EBV proteins that resembles the tumor necrosis factor (TNF) receptor and is essential for virus-induced cell signaling pathways. Thus, we can hypothesize that gC1q, in addition to CLR, is also involved in the EBV-induced autoimmune microenvironment. 
The waste-disposal hypothesis [38] appointed the clearance of apoptotic cells as a major event in triggering autoimmunity. C1q participates in that clearance by binding apoptotic cells directly via its gC1q [39,40]. Indirectly, C1q can also clear apoptotic cells bound by IgM [41-44]. The latter is a natural ligand of $\mathrm{Clq}$ for the activation of the classical complement pathway and the IgM-binding site is also located in gC1q [45]. Consequently, deficiency of $\mathrm{C} 1 \mathrm{q}$ results in impairment of apoptotic cell clearance which provides autoantigenic stimuli for autoreactive $B$ cells with diverse specificities $[46,47]$. The presence of anti-C1q antibodies could cause a functional deficiency of $\mathrm{C} 1 \mathrm{q}$. Anti-C1q autoantibodies from LN patients were reported to inhibit the clearance of apoptotic cells in vitro [48]. Given the apoptotic blebs are recognized by gC1q, it seems likely that the inhibiting autoantibodies are anti-gC1q. The revealed structural similarity of scFv A1 with the apical region of $\mathrm{gClq}$ opens up a possibility to test the hypothesis that anti-gC1q interferes with the mechanism of apoptotic cell clearance by $\mathrm{C} 1 \mathrm{q}$, both directly and indirectly, due to overlapping of the globular autoepitope with the apoptotic cell-binding site and/or IgM-binding site of C1q.

\section{Materials and Methods}

\subsection{Buffers}

The following buffers were used: sodium carbonate buffer (SC), pH 9.6; PBS containing $0.1 \%$ Tween 20 (TPBS); PBS with $0.75 \mathrm{M} \mathrm{NaCl}$ (PBS/0.75); elution buffer (EB) [0.1 M Glycine$\mathrm{HCl}, \mathrm{pH}$ 2.8]. 1-Anilinonaphthalene-8-sulfonic acid (ANS) was purchased from Sigma (St. Louis, MO, USA) and its concentration was determined spectrophotometrically by its extinction coefficient at $\lambda_{370} \mathrm{~nm}\left(\varepsilon_{370}=6.8 \times 10^{3} \mathrm{M}^{-1} \mathrm{~cm}^{-1}\right)$.

\subsection{Growth Media}

The following growth media were used: 2xTY medium (20 g/L Tryptone (Casitose Type-I, HiMedia, Mumbai, India), 10 g/L Yeast Extract (HiMedia, Mumbai, India), 20 g/L $\mathrm{NaCl}$ (Fisher Scientific, Loughborough, UK), pH 7.2) containing $100 \mu \mathrm{g} / \mathrm{mL}$ Ampicillin Ampicillin (Fisher Bioreagents, Pittsburg, PA, USA) and supplemented with $1 \mathrm{mM} \mathrm{MgSO}_{4}$ (Fisher Scientific, Loughborough, UK) and 1\% Glucose (Merck KGaA, Gernsheim, Germany) (2xTY-Amp); LB medium (10 g/L Tryptone, $5 \mathrm{~g} / \mathrm{L}$ Yeast Extract, $10 \mathrm{~g} / \mathrm{L} \mathrm{NaCl}$, $\mathrm{pH}$ 7.2) containing $100 \mu \mathrm{g} / \mathrm{mL}$ Ampicillin and supplemented with $1 \mathrm{mM} \mathrm{MgSO}_{4}$ and $1 \%$ Glucose (LB-Amp); ZYP-5052 medium for autoinduction (1\% Tryptone, $0.5 \%$ Yeast Extract, $25 \mathrm{mM}\left(\mathrm{NH}_{4}\right)_{2} \mathrm{SO}_{4}$ (Chem-Lab NV, Zedelgem, Belgium), $50 \mathrm{mM} \mathrm{KH} \mathrm{PO}_{4}$ (Fisher Bioreagents, UK, $50 \mathrm{mM} \mathrm{Na}{ }_{2} \mathrm{HPO}_{4}$ (Fisher Bioreagents, Loughborough, UK), 0.5\% glycerol (Sigma, St. Louis, MO, USA), 0.05\% glucose, $0.2 \% \alpha$-lactose (Carl Roth GmbH, Karlsruhe, Germany), $1 \mathrm{mM} \mathrm{MgSO}_{4}$ ), containing $100 \mu \mathrm{g} / \mathrm{mL}$ Ampicillin; TYE agar (10 g/l tryptone, $5 \mathrm{~g} / 1$ yeast, $8 \mathrm{~g} / 1 \mathrm{NaCl}, 15 \mathrm{~g} / 1$ agar), containing $100 \mu \mathrm{g} / \mathrm{mL}$ Ampicillin (TYE-Amp).

\subsection{Expression of the Globular Heads $g h A, g h B$ and $g h C$ Representing $g C 1 q$}

The recombinant $\mathrm{C} 1 \mathrm{q}$ globular heads ghA, ghB and ghC were expressed as fusion proteins with bacterial MBP in E. coli BL21 (DE3) and purified, as described previously [17].

\subsection{Preparation of Antigens for the Selection of Anti-Idiotypic scFv}

Six fractions of anti-C1q antibodies were affinity isolated from previously analyzed LN sera [15] according to their epitope specificity (Table 1). C1q-coated (4 $\mu \mathrm{g} /$ well) plates, blocked with $1 \%$ BSA, were incubated overnight at $4{ }^{\circ} \mathrm{C}$ with $250 \mu \mathrm{L} /$ well of pooled sera from groups A, B and C, diluted 1:50 in PBS/0.75. The wells were washed three times with TPBS and once with PBS. Bound anti-C1q was eluted by incubation of each well with $50 \mu \mathrm{L} /$ well EB for precisely five minutes and was immediately neutralized. Forty-eight C1q-coated wells were used for each group of pooled sera and the eluted $2.4 \mathrm{~mL}$ for every type of anti-C1q fractions were neutralized with $90 \mu \mathrm{L}$ of $1.5 \mathrm{M}$ Tris (Fisher Bioreagents, NJ, USA) ( $\mathrm{pH} 8.8$ ) and dialysed against PBS. 
Analogously, the pooled sera from groups D, E and F were incubated each on 48 ghCcoated wells (4 $\mathrm{g}$ /well), blocked with $1 \%$ BSA. This affinity isolation resulted in six anti$\mathrm{C} 1 \mathrm{q}$ fractions which were used as antigens for the selection of anti-idiotypic scFv antibodies.

\subsection{Screening of Human scFv Phagemid Library Griffin.1}

Panning of phage scFv antibodies was performed following [21]. Briefly, immunotubes (NUNC MaxiSorp) were coated overnight with $2.4 \mathrm{~mL}$ of each of the six antigen groups of anti-C1q preparations. The tubes were blocked with 0.5\% Tween 20 (Fisher Bioreagents, Fair Lawn, NJ, USA) for two hours and incubated with $1 \mathrm{~mL}$ (approximately $10^{13} \mathrm{PFU}$ ) of phage scFv in $0.5 \%$ Tween 20 for 30 min with rotation, followed by a stationary incubation for an additional $90 \mathrm{~min}$. All incubation steps were performed at room temperature. The tubes were washed 10 times with TPBS and 10 times with PBS. Bound phage scFv were eluted with $1 \mathrm{~mL}$ of $100 \mathrm{mM}$ triethylamine and gentle rotating of the tube for $10 \mathrm{~min}$. The eluted material was neutralized with $0.5 \mathrm{~mL} 1 \mathrm{M}$ Tris- $\mathrm{HCl}(\mathrm{pH}$ 7.4). Half of the eluted phage suspension was used to infect $10 \mathrm{~mL} \log$ phase E. coli TG1 cells at $37^{\circ} \mathrm{C}$ for $30 \mathrm{~min}$, and then plated on TYE-Amp plates for an overnight cultivation. The infected cells were transferred to $100 \mathrm{~mL}$ 2xTY-Amp and the log phase culture was co-infected with VCS-M13 helper phage and grown in 2xTY-Amp-Kan overnight at $30^{\circ} \mathrm{C}$. The rescued phage was precipitated with polyethylene glycol and used for the next round of selection. The library was subjected to three rounds of selection and resulted in producing sets of polyclonal phage for each antigen, e.g., anti-C1q fraction. Serial dilutions of all polyclonal phage were plated on TYE-Amp for titer determination.

\subsection{Selection of Monoclonal scFv}

The plates with serial dilutions of the polyclonal phage from the three rounds were used for selecting random monoclonal scFv phage for each of the six anti-C1q fractions. The selection was on the basis of colony morphology. The selected clones were inoculated into $100 \mu \mathrm{L} /$ well 2xTY-Amp in 96-well plates (Cell Well, Corning, Glendale, AZ, USA) and grown with shaking $\left(190 \mathrm{revs} / \mathrm{min}\right.$ ) overnight at $37^{\circ} \mathrm{C}$. The overnight cultures were transferred into a new 96-well plate by inoculation of $2 \mu \mathrm{L}$ in $200 \mu \mathrm{L}$ fresh 2xTY-Amp and cultivated for one hour. Then, $50 \mu \mathrm{L}$ of $2 \times$ TY-Amp containing $10^{10}$ PFU of VCS-Ml3 was added to each well and the plate was incubated at $37^{\circ} \mathrm{C}$ for $30 \mathrm{~min}$ without shaking. The cells were spun down by centrifugation at $3000 \mathrm{rev} / \mathrm{min}$ at $4{ }^{\circ} \mathrm{C}$, resuspended in $200 \mu \mathrm{L} /$ well $2 \times \mathrm{TY}$-Amp-Kan and grown overnight at $30^{\circ} \mathrm{C}, 120 \mathrm{rev} / \mathrm{min}$ shaking. The supernatant containing monoclonal phage was tested for binding of antigen by immunoblot. Phage that displayed $\mathrm{scFv}$ were incubated with antigen-coated nitrocellulose membranes. Bound phage was detected by anti-M13-HRP (Sigma-Aldrich, Darmstadt, Germany) and DAB (diaminobenzidine, Sigma-Aldrich, St. Louis, MO, USA).

\subsection{Induction of Soluble scFv and Affinity Purification}

The soluble scFv was induced by two alternative induction protocols [49]. Briefly, for IPTG induction, E. coli HB2151/A1 cultures $\left(\mathrm{OD}_{600 \mathrm{~nm}}\right.$ of 0.8$)$ were induced with $0.5 \mathrm{mM}$ IPTG (Fisher Bioreagents, UK) for five hours at $25^{\circ} \mathrm{C}$. For autoinduction, overgrown $E$. coli HB2151/A1 daily cultures were inoculated 1:100 in ZYP-5052 autoinduction medium and grown for $16 \mathrm{~h}$ at $25^{\circ} \mathrm{C}$. The induced cells were lysed in $1 / 20$ th of the culture volume in ice-cold $100 \mathrm{mM}$ Tris-HCl, pH.8.0, containing 20\% sucrose (Fisher Scientific, Loughborough, UK) and 1 mM EDTA (Fisher Bioreagents, Fair Lawn, NJ, USA) for one hour on ice and then centrifuged at $9000 \mathrm{rpm}$ for $45 \mathrm{~min}$ at $4{ }^{\circ} \mathrm{C}$. The yielded supernatant (SN1), containing soluble $\mathrm{scFv}$, was kept on ice and later pooled with $\mathrm{SN} 2$, obtained after the subsequent lysis of the cell pellet in the same volume of ice-cold $5 \mathrm{mM} \mathrm{MgSO}_{4}$ for $15 \mathrm{~min}$ on ice and then centrifuged at $9000 \mathrm{rpm}$ for $45 \mathrm{~min}$ at $4{ }^{\circ} \mathrm{C}$. The pooled supernatants were dialysed against phosphate buffer, pH 8.0 containing 10 mM Imidazole (Acros Organics, Fair Lawn, NJ, USA) and applied on HIS-Select ${ }^{\circledR}$ Ni-affinity gel column (Sigma-Aldrich, Saint Louis, $\mathrm{MO}, \mathrm{USA}$ ) at a flow rate of $0.5 \mathrm{~mL} / \mathrm{min}$. The $\mathrm{scFv}$ antibodies were eluted with phosphate 
buffer, $\mathrm{pH} 8.0$ containing $250 \mathrm{mM}$ Imidazole. The eluted protein samples were dialysed against PBS, pH 7.2.

\subsection{Enzyme-Linked Immunosorbent Assays}

The inhibitory ELISA analyses were performed with four test-antigens-the native C1q (Merck Millipore Calbiochem ${ }^{\mathrm{TM}}$ Calbiochem, Darmstadt, Germany) and the recombinant analogues of ghA, ghB and ghC. The source of autoantibodies, designated $\mathrm{IgG}_{\mathrm{LN}}$, was affinity purified IgG from pooled LN sera. Microtiter 96-flat bottom plates were coated with $\mathrm{Clq}$ or ghA, or ghB or ghC $(1 \mu \mathrm{g} /$ well in SC) and blocked with $1 \%$ BSA (Fisher Scientific, UK). Increasing amounts of the analysed clone $\operatorname{scFv}(2.5,5$ and $10 \mu \mathrm{g} /$ well) was pre-incubated with biotinylated $\mathrm{IgG}_{\mathrm{LN}}$ in $\mathrm{PBS} / 0.75$ for $30 \mathrm{~min}$ at room temperature and then added to the antigen-coated plates for an overnight incubation at $4{ }^{\circ} \mathrm{C}$. Bound autoantibodies were detected by ExtrAvidin-AP (Sigma-Aldrich, St. Louis, MO, USA) and para-nitrophenylphosphate (pNPP, Life Technologies, Inc. Gaithersburg, MD, USA). The absorbance was read at $405 \mathrm{~nm}$ with a microplate reader (DR-200B, Hiwell Diatek Instruments, Wuxi, China). All samples were analysed in triplicate.

\subsection{Spectroscopy Study of the Binding of Native C1q and ghA, ghB and ghC (Recombinant Fragments)}

Emission spectra were registered by a Shimadzu spectrofluorophotometer RF 5000 (Shimadzu, Tokyo, Japan). The fluorescence spectroscopy experiments were performed in a $1 \mathrm{~cm}$ quartz cell (Hellma Analytics, Müllheim, Germany). C1q, ghA, ghB and ghC were studied using fluorescent dye ANS, excited at $390 \mathrm{~nm}$ with a slit width of $1.0 \mathrm{~nm}$. Total fluorescence was calculated after normalizing the spectra and correcting for dilution. For the normalization of the inner filter and self-absorption effects, the excitation was always carried out less than $0.05 \mathrm{OD}$. Studies were done at $22^{\circ} \mathrm{C}$. The binding of $\mathrm{C} 1 \mathrm{q}$ and ghA, ghB and ghC with increasing concentrations $(0.03-0.3 \mu \mathrm{M})$ were added into the cuvette, containing LN autoantibodies complexed with scFv A1.

\subsection{Sequence Analysis of Selected Clones}

ScFv A1 coding sequence in pHEN2 vector was amplified with in situ polymerase chain reaction (PCR) of single colony E. coli HB2151, primers LMB3 (5'-CAGGAAACAGCTATGAC-3') and fd-SEQ1 (5'-GAATTTTCTGTATGAGG-3'), according to amplification conditions of Liners et al. [50]. The PCR product was purified (GE Healthcare illus$\operatorname{tra}^{\mathrm{TM}}$ GFX ${ }^{\mathrm{TM}}$ PCR DNA and Gel Band Purification Kit) and further sent for bidirectional Sanger sequencing (Macrogen, Amsterdam, the Netherlands). The derived sequence was manually aligned, cured from vector parts and deposited in GenBank under accession number KX981596.

\subsection{1. scFv A1 Homology Model Generation}

The MOE2016 software [51] was used for the homology modeling. Crystallized structures used for our homology modeling were derived from the Protein Data Bank (PDB). We performed alignment of the target sequence to the selected parts of best scored XRD structures of antibodies according to the modified version of the Needleman and Wunsch algorithm [52] with sequence alignment imposed on amino acid BLOSUM 62 substitution matrix, with algorithm penalty for gap opening -12 and gap extension -2 and evaluated with E-value accepted 1.e -12. The first five hits (PDB ID: 1YNL, 1A5F, 1ETZ, 1DEE, 1AD9) showing statistically significant similarity, the most similar one having $\mathrm{E} \sim 2 . \mathrm{e}-42$, were chosen for further structure modeling of our sequence.

The homology model of scFv A1 was carried out by the Boltzmann-weighted randomized modeling procedure using coulomb and generalized Born implicit solvent interaction energies, using MMFF94 force field, as was implemented in MOE software suite (MOE2016).

The obtained 3D structure of scFv A1 sequence by homology modeling was further relaxed by molecular dynamics simulations with GROMACS software [53]. The structure of scFv A1 was neutralized and placed in periodic boundary conditions with explicit water 
molecules (TIP3P) with added $\mathrm{Cl}^{-}$and $\mathrm{Na}^{+}$ions up to physiological concentration of $0.154 \mathrm{M} / \mathrm{L}$. The simulation was performed with CHARMM27 force field and the final production run was done on an NPT ansembl, 300K, 1atm, Berendsen thermostat and Parrinello-Rahman pressostat. The last $100 \mathrm{~ns}$ of equilibrated in physiological condition structure was used for further analysis. The selected trajectory part was used for cluster analysis and the frame closest to the center of the largest cluster was used further.

\subsection{C1q Modeling}

PDB structure was checked and several structural improperties were corrected. Other non-protein species were removed. The missing amino acids were placed according to the electron density map of the structures, despite the low electron densities there, with the COOT 0.8.8 software [54]. New protons were added in order to achieve right protonation state at $7.0 \mathrm{pH}$ as the experimental study was carried out at physiological conditions. Further molecular dynamics simulation and analysis were done as in the case of the $\mathrm{scFv}$ A1 model.

\subsection{Comparative Analysis of scFv A1 Homology Model and ghC1q Chains}

Sequence comparison of modelled globular head domains of human $\mathrm{C} 1 \mathrm{q}$ and $\mathrm{H}-$ chain of generated homology model of scFv A1 was performed, using secondary structure information of those sequences having associated atomic coordinates. The target sequence was sequence-to-group aligned to each one of ghC1q chains. Manual alignment was used for refining comparison of CDR frames of scFv A1 H-chain and loops in A-, B- and C- ghC1q.

\section{Conclusions}

We generated an anti-idiotypic scFv, clone A1, which inhibited approximately $50 \%$ the recognition of human $\mathrm{Clq}$ and its globular fragments ghA, ghB and ghC by the LN autoantibodies. The CDRs of $\mathrm{scFv}$ A1 showed structural similarity to the apical region of $\mathrm{gClq}$ comprising parts of all globular fragments thus localizing a globular autoepitope of $\mathrm{C} 1 \mathrm{q}$ of a conformational type.

The $\mathrm{V}_{\mathrm{H}} \mathrm{CDR} 2$ of $\mathrm{A} 1$ mimics the ghA sequence GSEAD suggested as a cross-epitope between anti-DNA and anti-C1q antibodies.

Other potential structural analogs to $\mathrm{C} 1 \mathrm{q}$ autoepitopes are the inhibitory monoclonal scFv F6, F9 and A12.

Supplementary Materials: The following are available online at https://www.mdpi.com/article/ $10.3390 / \mathrm{ijms} 22158288 / \mathrm{s} 1$. Table S1. Data representing the plot in Figure 1 for each of the partially purified anti-idiotypic scFv clones analysed as inhibitors of the recognition of immobilised C1q by $\mathrm{IgG}_{\mathrm{LN}}$ with $\mathrm{SD}$ values.

Author Contributions: Conceptualization, I.T.; Data curation, N.T. and M.R.; Formal analysis, N.T., M.R., V.B., V.S., A.Y., G.N., H.G., D.D., S.M., K.S. and I.T.; Investigation, N.T., M.R., V.B., V.S., A.Y., G.N., H.G., D.D., S.M. and K.S.; Methodology, N.T., M.R., V.B., V.S., G.N., H.G., D.D., S.M., K.S. and I.T.; Project administration, I.T.; Resources, M.R. and V.B.; Writing—original draft, I.T.; Writingreview \& editing, N.T., M.R., V.B., V.S., A.Y., G.N., H.G., D.D., S.M. and K.S. All authors have read and agreed to the published version of the manuscript.

Funding: This research was funded by the Bulgarian NSF, grant number DN01/9.

Institutional Review Board Statement: The study was conducted according to the guidelines of the Declaration of Helsinki, and approved by the Ethics Committee of Sofia University (protocol code 93-И-115/14 June 2019).

Informed Consent Statement: Informed consent from all subjects involved in the study was obtained previously for the study published at https://doi.org/10.1016/j.imlet.2012.08.007 (accessed on 14 June 2019). 
Data Availability Statement: The data that support the findings of this study are available from the corresponding author upon reasonable request.

Conflicts of Interest: The authors declare no conflict of interest.

\section{References}

1. Agnello, V.; Koffler, D.; Eisenberg, J.W.; Winchester, R.J.; Kundel, H.G. C1q precipitins in the sera of patients with systemic lupus erythematosus and other hypocomplementemic states: Characterization of high and low molecular weight types. J. Exp. Med. 1971, 134, 228-241. [CrossRef] [PubMed]

2. Daha, M.R. Pathogenic role of auto-antibodies against complement components in systemic lupus erythematosus. Lupus 2008, 17, 385-388. [CrossRef]

3. Potlukova, E.; Kralikova, P. Complement Component C1q and Anti-C1q Antibodies in Theory and in Clinical Practice. Scand. J. Immunol. 2008, 67, 423-430. [CrossRef]

4. Rahman, A.; Isenberg, D.A. Systemic Lupus Erythematosus. N. Engl. J. Med. 2008, 358, 929-939. [CrossRef]

5. Rahman, A.; Hiepe, F. Anti-DNA antibodies-overview of assays and clinical correlations. Lupus 2002, 11, 770-773. [CrossRef]

6. Golan, M.D.; Burger, R.; Loos, M. Conformational changes in C1q after binding to immune complexes: Detection of neoantigens with monoclonal antibodies. J. Immunol. 1982, 129, 445-447.

7. Trouw, L.A.; Groeneveld, T.W.; Seelen, M.A.; Duijs, J.M.; Bajema, I.M.; Prins, F.A.; Kishore, U.; Salant, D.J.; Verbeek, J.S.; van Kooten, C.; et al. Anti-C1q autoantibodies deposit in glomeruli but are only pathogenic in combination with glomerular C1q-containing immune complexes. J. Clin. Investig. 2004, 114, 679-688. [CrossRef]

8. Bigler, C.; Schaller, M.; Perahud, I.; Trendelenburg, M. Autoantibodies against complement C1q specifically target C1q bound on early apoptotic cells. Mol. Immunol. 2009, 183, 3512-3521. [CrossRef] [PubMed]

9. Stoyanova, V.; Bogoeva, V.; Petrova, L.; Tchorbadjieva, M.; Petrova, S.; Georgieva, V.; Georgiev, G.; Deliyska, B.; Vasilev, V.; Tsacheva, I. Autoantigenicity of human C1q is associated with increased hydrophobicity due to conformational transitions in the globular heads. Mol. Bio. Syst. 2015, 11, 1370-1377. [CrossRef] [PubMed]

10. Eggleton, P.; Ukoumunne, O.C.; Cottrell, I.; Khan, A.; Maqsood, S.; Thornes, J.; Perry, E.; Isenberg, D. Autoantibodies against C1q as a diagnostic measure of lupus nephritis: Systematic review and meta-analysis. J. Clin. Cell Immunol. 2014, 5. [CrossRef]

11. Seshan, S.V.; Jennette, J.C. Renal disease in systemic lupus erythematosus with emphasis on classification of lupus glomerulonephritis: Advances and implications. Arch. Pathol. Lab. Med. 2009, 133, 233-248. [CrossRef]

12. Ortega, L.M.; Schultz, D.R.; Lenz, O.; Pardo, V.; Contreras, G.N. Review: Lupus nephritis: Pathologic features, epidemiology and a guide to therapeutic decisions. Lupus 2010, 19, 557-574. [CrossRef]

13. Trendelenburg, M. Antibodies against C1q in patients with systemic lupus erythematosus. Springer Semin. Immunopathol. 2005, 27, 276-285. [CrossRef]

14. Schaller, M.; Bigler, C.; Danner, D.; Ditzel, H.J.; Trendelenburg, M. Autoantibodies against C1q in systemic lupus erythematosus are antigen-driven. J. Immunol. 2009, 183, 8225-8231. [CrossRef] [PubMed]

15. Stoyanova, V.; Tchorbadjieva, M.; Deliyska, B.; Vasilev, V.; Tsacheva, I. Biochemical analysis of the epitope specificities of anti-C1q autoantibodies accompanying human Lupus Nephrites reveals them as a dynamic population in the course of the disease. Immunol. Lett. 2012, 148, 69-76. [CrossRef]

16. Wener, M.H.; Uwatoko, S.; Mannik, M. Antibodies to the collagen-like region of C1q in sera of patients with autoimmune rheumatic diseases. Arth. Rheum. 1989, 32, 544-551. [CrossRef]

17. Tsacheva, I.; Radanova, M.; Todorova, N.; Argirova, T.; Kishore, U. Detection of autoantibodies against the globular domain of human C1q in the sera of systemic lupus erythematosus patients. Mol. Immunol. 2007, 44, 2157-2161. [CrossRef]

18. Tan, Y.; Zhou, W.; Yu, F.; Fang, Q.; Yang, H.-z.; Zhao, M.-h. Detection of anti-C1q antibodies and anti-C1q globular head domain antibodies in sera from Chinese patients with lupus nephritis. Mol. Immunol. 2009, 46, 2178-2182. [CrossRef]

19. Vanhecke, D.; Roumenina, L.T.; Wan, H.; Osthoff, M.; Schaller, M.; Trendelenburg, M. Identification of a major linear C1q epitope allows detection of systemic lupus erythematosus anti-C1q antibodies by a specific peptide-based enzyme-linked immunosorbent assay. Arth. Rheum. 2012, 64, 3706-3714. [CrossRef] [PubMed]

20. Kishore, U.; Ghai, R.; Greenhough, T.J.; Shrive, A.K.; Bonifati, D.M.; Gadjeva, M.G.; Waters, P.; Kojouharova, M.S.; Chakraboty, T.; Agrawal, A. Structural and functional anatomy of the globular domain of complement protein C1q. Immunol. Lett. 2004, 95, 113-128. [CrossRef] [PubMed]

21. Marks, J.D.; Hoogenboom, H.R.; Bonnert, T.P.; McCafferty, J.; Griffiths, A.D.; Winter, G. By-passing immunization: Human antibodies from V-gene libraries displayed on phage. J. Mol. Biol. 1991, 222, 581-597. [CrossRef]

22. Retter, I.; Althaus, H.H.; Münch, R.; Müller, W. VBASE2, an integrative V gene database. Nucleic Acids Res. 2005, 33 (Suppl. S1), D671-D674. [CrossRef]

23. Gaboriaud, C.; Juanhuix, J.; Gruez, A.; Lacroix, M.; Darnault, C.; Pignol, D.; Verger, D.; Fontecilla-Camps, J.C.; Arlaud, G.J. The crystal structure of the globular head of complement protein $\mathrm{C} 1 \mathrm{q}$ provides a basis for its versatile recognition properties. J. Biol. Chem. 2003, 278, 46974-46982. [CrossRef] [PubMed]

24. Franchin, G.; Son, M.; Kim, S.J.; Ben-Zvi, I.; Zhang, J.; Diamond, B. Anti-DNA antibodies cross-react with C1q. J. Autoimmun. 2013, 44, 34-39. [CrossRef] [PubMed] 
25. Mahler, M.; van Schaarenburg, R.A.; Trouw, L.A. Anti-C1q autoantibodies, novel tests and clinical consequences. Front. Immunol. 2013, 4, 117. [CrossRef] [PubMed]

26. Moroni, G.; Trendelenburg, M.; Del Papa, N.; Quaglini, S.; Raschi, E.; Panzeri, P.; Testoni, C.; Tincani, A.; Banfi, G.; Balestrieri, G.; et al. Anti-C1q antibodies may help in diagnosing a renal flare in lupus nephritis. Am. J. Kidney Dis. 2001, 37, 490-498. [CrossRef]

27. Trendelenburg, M.; Lopez-Trascasa, M.; Potlukova, E.; Moll, S.; Regenass, S.; Fremeaux-Bacchi, V.; Martinez-Ara, J.; Jancova, E.; Picazo, M.L.; Honsova, E.; et al. High prevalence of anti-C1q antibodies in biopsy-proven active lupus nephritis. Nephrol. Dial. Transpl. 2006, 21, 3115-3121. [CrossRef] [PubMed]

28. Moura, C.G.; Lima, I.; Barbosa, L.; Athanazio, D.; Reis, E.; Reis, M.; Burlingame, R.W.; Santiago, M.B. Anti-C1q Antibodies: Association with Nephritis and Disease Activity in Systemic Lupus Erythematosus. J. Clin. Lab. Anal. 2009, 23, 19-23. [CrossRef]

29. Akhter, E.; Burlingame, R.; Seaman, A.; Magder, L.; Petri, M. Anti-C1q antibodies have higher correlation with flares of lupus nephritis than other serum markers. Lupus 2011, 20, 1267-1274. [CrossRef]

30. Yin, Y.; Wu, X.; Shan, G.; Zhang, X. Diagnostic value of serum anti-C1q antibodies in patients with lupus nephritis: A meta-analysis. Lupus 2012, 21, 1088-1097. [CrossRef] [PubMed]

31. Wener, M.H. Autoantibodies to C1q. In Autoantibodies; Elsevier: Oxford, UK, 2014; pp. 707-715.

32. Stoyanova, V.; Petrova, S.; Tchorbadjieva, M.; Deliyska, B.; Vasilev, V.; Tsacheva, I. New insight into the autoimmunogenicity of the complement protein C1q. Mol. Immunol. 2011, 48, 678-682. [CrossRef] [PubMed]

33. Rosen, A.; Casciola-Rosen, L. Autoantigens as partners in initiation and propagation of autoimmune rheumatic diseases. Ann. Rev. Immunol. 2016, 34, 395-420. [CrossRef]

34. Arbuckle, M.R.; McClain, M.T.; Rubertone, M.V.; Scofield, R.H.; Dennis, G.J.; James, J.A.; Harley, J.B. Development of autoantibodies before the clinical onset of systemic lupus erythematosus. N. Engl. J. Med. 2003, 349, 1526-1533. [CrossRef] [PubMed]

35. Eriksson, C.; Kokkonen, H.; Johansson, M.; Hallmans, G.; Wadell, G.; Rantapää-Dahlqvist, S. Autoantibodies predate the onset of systemic lupus erythematosus in northern Sweden. Arthritis Res. Ther. 2011, 13, R30. [CrossRef]

36. Csorba, K.; Schirmbeck, L.A.; Tuncer, E.; Ribi, C.; Roux-Lombard, P.; Chizzolini, C.; Huynh-Do, U.; Vanhecke, D.; Trendelenburg, M. Anti-C1q antibodies as occurring in systemic lupus erythematosus could be induced by an epstein-barr virus-derived antigenic site. Front. Immunol. 2019, 10, 2619. [CrossRef] [PubMed]

37. Fang, C.Y.; Chiang, C.Y.; Pan, Y.R.; Tse, K.P.; Chang, Y.S.; Chang, H.Y. Modulation of Epstein-Barr virus latent membrane protein 1 activity by intrabodies. Intervirology 2007, 50, 254-263. [CrossRef]

38. Botto, M.; Walport, M.J. C1q, autoimmunity and apoptosis. Immunobiology 2002, 205, 395-406. [CrossRef]

39. Navratil, J.S.; Watkins, S.C.; Wisnieski, J.J.; Ahearn, J.M. The globular heads of C1q specifically recognize surface blebs of apoptotic vascular endothelial cells. J. Immunol. 2001, 166, 3231-3239. [CrossRef] [PubMed]

40. Nauta, A.J.; Trouw, L.A.; Daha, M.R.; Tijsma, O.; Nieuwland, R.; Schwaeble, W.J.; Gingras, A.R.; Mantovani, A.; Hack, E.C.; Roos, A. Direct binding of C1q to apoptotic cells and cell blebs induces complement activation. Eur. J. Immunol. 2002, 32, 1726-1736. [CrossRef]

41. Zwart, B.; Ciurana, C.; Rensink IManoe, R.; Hack, C.E.; Aarden, L.A. Complement activation by apoptotic cells occurs predominantly via IgM and is limited to late apoptotic (secondary necrotic) cells. Autoimmunity 2004, 37, 95-102. [CrossRef]

42. Quartier, P.; Potte, P.K.; Ehrenstein, M.R.; Walport, M.J.; Botto, M. Predominant role of IgM-dependent activation of the classical pathway in the clearance of dying cells by murine bone marrow-derived macrophages in vitro. Eur. J. Immunol. 2005, 35, 252-260. [CrossRef]

43. Ogden, C.A.; Kowalewski, R.; Peng, Y.; Montenegro, V.; Elkon, K.B. IgM is required for efficient complement mediated phagocytosis of apoptotic cells in vivo. Autoimmunity 2005, 38, 259-264. [CrossRef]

44. Chen, Y.; Park, Y.B.; Patel, E.; Silverman, G.J. IgM antibodies to apoptosis-associated determinants recruit C1q and enhance dendritic cell phagocytosis of apoptotic cells. J. Immunol. 2009, 182, 6031-6043. [CrossRef]

45. Kojouharova, M.S.; Tsacheva, I.G.; Tchorbadjieva, M.I.; Reid, K.B.; Kishore, U. Localization of ligand-binding sites on human $\mathrm{C} 1 \mathrm{q}$ globular head region using recombinant globular head fragments and single-chain antibodies. Biochim. Et Biophys. Acta (BBA)-Proteins Proteom. 2003, 1652, 64-74. [CrossRef]

46. Botto, M.; Dell'Agnola, C.; Bygrave, A.E.; Thompson, E.M.; Cook, H.T.; Petry, F.; Loos, M.; Pandolfi, P.P.; Walport, M.J. Homozygous C1q deficiency causes glomerulonephritis associated with multiple apoptotic bodies. Nat. Genet. 1998, 19, 56-59. [CrossRef] [PubMed]

47. Fossati-Jimack, L.; Cortes-Hernandez, J.; Norsworthy, P.J.; Walport, M.J.; Cook, H.T.; Botto, M. C1q deficiency promotes the production of transgenic-derived IgM and IgG3 autoantibodies in anti-DNA knock-in transgenic mice. Mol. Immunol. 2008, 45, 787-795. [CrossRef] [PubMed]

48. Pang, Y.; Yang, X.W.; Song, Y.; Yu, F.; Zhao, M.H. Anti-C1q autoantibodies from active lupus nephritis patients could inhibit the clearance of apoptotic cells and complement classical pathway activation mediated by C1q in vitro. Immunobiology 2014, 219, 980-989. [CrossRef]

49. Nikolova, G.; Georgieva, Y.; Atanasova, A.; Radulova, G.; Kapogianni, A.; Tsacheva, I. Autoinduction as Means forOptimization of the Heterologous Expression of Recombinant Single-Chain Fv (scFv) Antibodies. Mol. Biotechnol. 2021. [CrossRef] [PubMed]

50. Liners, F.; Helbert, W.; Van Cutsem, P. Production and characterization of a phage-display recombinant antibody against carrageenans: Evidence for the recognition of a secondary structure of carrageenan chains present in red algae tissues. Glycobiology 2005, 15, 849-860. [CrossRef]

51. MOE (Molecular Operating Environment) Version 20170530; Chemical Computing Group ULC: Montreal, QC, Canada, 2018. 
52. Needleman, S.B.; Wunsch, C.D. A general method applicable to the search for similarities in the amino acid sequence of two proteins. J Mol Biol. 1970, 48, 443-453. [CrossRef]

53. GROMACS version 2016.5. In Software Versatile Package to Perform Molecular Dynamics; free software; University of Groningen: Groningen, Netherlands; Royal Institute of Technology: Stockholm, Sweden, Uppsala University: Netherlands, Sweden; 1991.

54. Emsley, P. COOT Version 0.8.8 Software, Crystallographic Object-Oriented Toolkit; Free Software; MRC Laboratory of Molecular Biology: Cambridge, UK, 2004. 